\title{
Caracterización mecánica por ondas de esfuerzo de la madera laminada de tres especies angiospermas
}

\author{
Javier Ramón Sotomayor Castellanos \\ Universidad Michoacana de San Nicolás de Hidalgo, Morelia, México.
}

\begin{abstract}
RESUMEN Existe evidencia empírica de que la madera laminada, comparativamente con la madera sólida mejora su densidad aparente y su módulo de elasticidad. El objetivo de la investigación fue determinar la densidad, la velocidad de onda y el módulo dinámico por ondas de esfuerzo en madera sólida y laminada de Enterolobium cyclocarpum, Tabebuia rosea y Juglans pyriformis. Se elaboraron probetas de madera sólida y laminada de pequeñas dimensiones y se realizaron pruebas de ondas de esfuerzo. La densidad aparente de la madera laminada, en comparación con la de la madera sólida, se incrementa para las tres especies. En cambio, el módulo dinámico de la madera laminada aumenta para Enterolobium cyclocarpum y Tabebuia rosea, pero disminuye para Juglans pyriformis. La variabilidad de la densidad aparente, la velocidad de onda y el módulo dinámico, aumentan o disminuyen según se trate de la especie y o del parámetro en cuestión. Los resultados son válidos para el caso de laminado de pequeñas probetas de las especies estudiadas. Para su aplicación a proyectos de ingeniería, es recomendable realizar estudios ex profeso.
\end{abstract}

Palabras clave: densidad aparente; velocidad de onda; módulo dinámico.

\section{Mechanical characterization by stress waves of the laminated wood of three angiosperm species}

\begin{abstract}
There is empirical evidence that laminated wood, compared to solid wood, improves its bulk density and modulus of elasticity. The objective of the research was to determine the apparent density, the wave velocity and the dynamic modulus by stress waves in solid and laminated wood of Enterolobium cyclocarpum, Tabebuia rosea and Juglans pyriformis. Solid and laminated wood specimens of small dimensions were prepared, and stress wave tests were carried out. The apparent density of laminated wood, in comparison with that of solid wood, increases for all three species. In contrast, the dynamic modulus of laminated wood increases for Enterolobium cyclocarpum and Tabebuia rosea but decreases for Juglans pyriformis. The variability of the apparent density, the wave velocity and the dynamic modulus, increases or decreases depending on the species and/or parameter in question. The results are valid for the case of laminated small specimens of the species studied. For its application to engineering projects, it is advisable to carry out ex profeso studies.
\end{abstract}

Keywords: apparent density; wave speed; dynamic module.

\section{Introducción}

La tecnología de modificación de la madera se ha implementado para mejorar sus propiedades intrínsecas y adquirir la forma y la funcionalidad requeridas por el diseño ingenieril (SANDBERG et al., 2017). Entre estas técnicas, el laminado de la madera es un procedimiento para transformar la geometría natural del árbol, en elementos con forma de paralelepípedos que se pueden usar en ingeniería (WALKER, 2006). Dos de los objetivos principales de esta tecnología son reducir la variabilidad natural de la estructura de la madera e incrementar su densidad aparente. El primer objetivo mejora el aprovechamiento de piezas pequeñas de material, las cuales se pueden conformar en piezas de amplias dimensiones y/o 
geometrías (WILLIAMSON, 2001). Dado que la densidad aparente de la madera es un buen descriptor de sus propiedades mecánicas (NIKLAS; SPATZ, 2010), el segundo objetivo pretende aumentar la resistencia mecánica al incrementar la densidad aparente de la madera sólida, al transformarla en madera laminada.

La densidad aparente de la madera es la medida de la masa de la madera por unidad de volumen y se mide a un contenido de humedad especificado, cuya masa está comprendida en el cálculo (WILLIAMSON; WIEMANN, 2010). Este parámetro es predictor del módulo dinámico de la madera y se emplea como clasificador de su calidad (MANSFIELD et al. 2016).

Las pruebas de ondas de esfuerzo son empleadas para medir el tiempo de transmisión de una onda a través de la dirección longitudinal de una pieza de madera. De manera no invasiva, al ponderar el tiempo por la distancia de recorrido, es posible determinar la velocidad de onda. De tal forma, que la combinación de la densidad aparente y de la velocidad de onda, permiten el cálculo del módulo dinámico de piezas de madera (BAYESTORFF; MONTEIRO, 2011).

Enterolobium cyclocarpum (Jacq.) Griseb., Tabebuia rosea (Bertol.) DC. y Juglans pyriformis Liebm, son especies endémicas de México y Centroamérica (CORDERO; BOSHIER, 2003). Estas especies son apreciadas para la fabricación de productos de madera con alto valor agregado. Sus características tecnológicas están referidas por Tamarit; López (2007), Silva et al. (2010) y Sotomayor (2015). Respecto a otras especies, De Souza et al. (2011), Bayestorff; Monteiro (2011), Del Menezzi et al. (2013) y Neto et al. (2016) han determinado el módulo dinámico con ondas de esfuerzo de madera laminada de diferentes especies. Sin embargo, no se detectó información de las características mecánicas de madera laminada de estas especies, particularmente determinadas por ondas de esfuerzo.
Existe evidencia empírica de que la madera laminada, comparativamente con la madera sólida, mejora su densidad aparente y su módulo de elasticidad (ERDIL et al, 2009). Esta tecnología reduce además defectos naturales en la madera sólida, como son nudos y fisuras estructurales (NADIR; NAGARAJAN, 2014). Así, la madera laminada puede ser una alternativa para sustituir a la madera sólida (BOURREAU et al, 2013). La hipótesis de la investigación es que el tratamiento de laminado incrementa la densidad aparente y el módulo dinámico de la madera. Esta propuesta está restringida a las especies estudiadas y para el caso de probetas de pequeñas dimensiones.

El objetivo de la investigación fue determinar la densidad, la velocidad de onda y el módulo dinámico por ondas de esfuerzo en madera sólida y laminada de Enterolobium cyclocarpum, Tabebuia rosea y Juglans pyriformis.

\section{Material y métodos}

Se estudiaron tres especies: Enterolobium cyclocarpum, Tabebuia rosea y Juglans piriformes, y se efectuaron pruebas con 35 réplicas (probetas por especie), recortadas al azar de piezas comerciales de madera aserrada. Para su análisis se observaron los resultados especie por especie, como muestras independientes, totalizando 105 observaciones para cada una de las variables.

El tratamiento de laminado de madera se consideró el factor de variación, de esa forma, se definieron dos niveles (estados de la madera): madera sólida (MS) y madera laminada (ML). Las variables de respuesta para MS y ML fueron la densidad aparente $\left(\rho_{\mathrm{CH}}\right)$ la velocidad de onda $\left(\mathrm{v}_{\mathrm{oe}}\right)$ y el módulo dinámico $\left(\mathrm{E}_{\mathrm{oe}}\right)$. Las dimensiones de las probetas $\mathrm{y}$ el contenido de humedad, se consideraron factores controlables y con variación mínima, de tal forma que no intervienen en el fenómeno observado. La variabilidad en la estructura anatómica y en la composición de la madera 
laminada, se consideraron factores que tampoco influyeron en el fenómeno.

Para todas las variables se calcularon la media $(\overline{\mathrm{x}})$, la desviación estándar $(\sigma)$, y el coeficiente de variación $(\mathrm{CV}=$ $\overline{\mathrm{x}} / \sigma)$. Se realizaron pruebas de normalidad en la distribución de los datos de cada muestra. El criterio de demarcación fue aceptar los valores calculados del sesgo estandarizado (SE), y del apuntamiento estandarizado (AE), al interior del intervalo $[-2,+2]$.

Una vez confirmada la normalidad, el experimento consistió en la verificación de la varianza (Ver-var), y su análisis (Anova), cuando no se verificó la normalidad de las muestras, se procedió a realizar pruebas no paramétricas de Kruskal-Wallis (K-W) de diferencia de medianas. Todas las pruebas fueron para un nivel del 95\% de confianza. El criterio de demarcación para las pruebas Anova y K-W, fue aceptar una diferencia estadísticamente significativa para un valor $\mathrm{P}$ - 0,05 .

No obstante que el número de probetas por cada muestra fue de 35 , cantidad suficiente para considerar la teoría de las grandes muestras en el análisis, se calculó a posteriori el tamaño de la muestra necesario para validar las pruebas estadísticas. El tamaño de la muestra (número de probetas), se calculó con la ecuación (1) (GUTIÉRREZ; DE LA VARA, 2012):

$$
\mathrm{n}=\frac{2 \sigma^{2}}{\mathrm{e}^{2}}
$$

Donde: $\mathrm{n}=$ Tamaño de la muestra; $\sigma=$ Desviación estándar; e = Error de estimación aceptable.

Un segundo enfoque en la estrategia experimental fue el considerar como una sola muestra a los 175 resultados de cada una de las variables de respuesta de las tres especies. Así, se determinaron las regresiones lineales $(y=a x \pm b)$, y su coeficiente de determinación $\left(\mathrm{R}^{2}\right)$, para la velocidad de onda $\left(\mathrm{v}_{\mathrm{oe}}\right)$, y el módulo dinámico $\left(\mathrm{E}_{\mathrm{oe}}\right)$, como variables dependientes de la densidad aparente $\left(\rho_{\mathrm{CH}}\right)$, como variable independiente.

Las probetas se elaboraron a partir de piezas de madera aserrada adquiridas en empresas comercializadoras de maderas, en el Estado de Michoacán, México. El taxón botánico fue identificado en el Laboratorio de Mecánica de la Madera, de la Universidad Michoacana de San Nicolás de Hidalgo, en Morelia, Michoacán. Para cada una de las tres especies, se prepararon dos grupos de probetas de acuerdo a la norma ISO 3129:2012 (Internacional Organization for Standardization, 2012), con dimensiones de 0,02 m x 0,02 m x $0,4 \mathrm{~m}$, correspondientes a las direcciones radial, tangencial y longitudinal de la madera.

Las probetas se almacenaron en una cámara de acondicionamiento con temperatura de $20^{\circ} \mathrm{C}\left( \pm 1^{\circ} \mathrm{C}\right)$ y con humedad relativa del aire de $65 \%( \pm 2 \%)$, hasta que alcanzaron un contenido de humedad en equilibrio de $10 \%$. El contenido de humedad de la madera $(\mathrm{CH})$, fue calculado por el método de diferencias del peso de las probetas al momento del ensayo en relación a su peso anhidro (ISO 13061-1:2014, Internacional Organization for Standardization, 2014a). La densidad aparente de la madera $\left(\rho_{\mathrm{CH}}\right)$, se determinó con la relación peso de la probeta y su volumen, ambas medidas realizadas al momento del ensayo (ISO 13061-2:2014, Internacional Organization for Standardization, 2014b).

Para la fabricación de las probetas de madera laminada, se utilizaron cinco tabletas de madera sólida, de acuerdo a la estructura sugerida por Sotomayor; Ruiz (2017). Para adherir las tabletas, se aplicaron $2,5 \mathrm{~kg} \mathrm{~m}^{-2}$ de pegamento de contacto de acetato de vinilo. Una vez formadas las probetas, se posicionaron en un dispositivo ad-hoc y se prensaron hasta que las probetas alcanzaron una altura y/o espesor uniforme de 0,02 m. Con el objetivo de que el adhesivo solidificara, el tiempo de prensado fue de 48 horas en ambiente de 
laboratorio (Temperatura de $20^{\circ} \mathrm{C}$ y humedad relativa del aire de $65 \%)$. Finalmente, las probetas de madera laminada, se almacenaron durante tres meses en la cámara de acondicionamiento con las condiciones antes citadas de temperatura y de humedad relativa del aire, hasta que su peso fue constante y recuperaran un contenido de humedad en equilibrio de $10 \%$.

Las pruebas de ondas de esfuerzo siguieron el protocolo reportado por Sotomayor; Ruiz (2017), y consistieron en suministrar un impacto en un extremo de la probeta y provocar una onda de esfuerzo a través de la dirección longitudinal y registrar el tiempo de transmisión necesario para que la onda llegue al extremo opuesto de la probeta. Para tal propósito, se utilizó el aparato Metriguard ${ }^{\circledR}$ posicionado en un dispositivo para pruebas no destructivas diseñado $a d-$ hoc (Figura 1). Con la longitud de las probetas y el tiempo de transmisión de las ondas de esfuerzo, se calculó su velocidad.

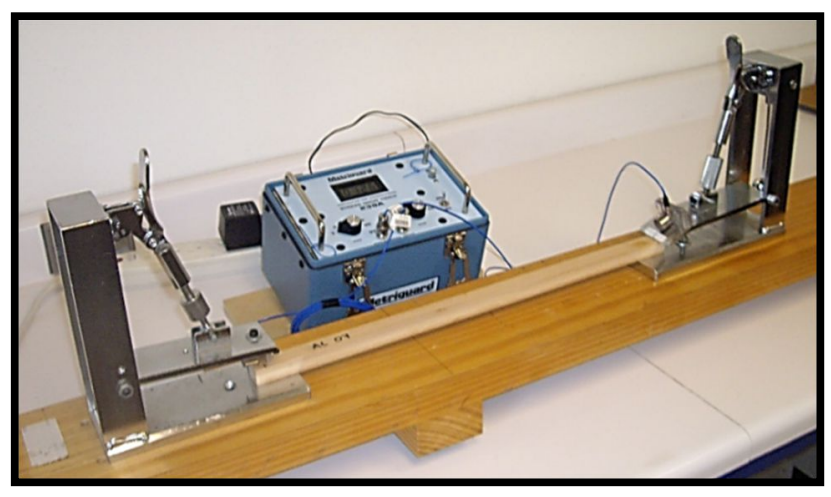

Figura 1. Pruebas de ondas de esfuerzo.

El módulo de elasticidad dinámico se calculó con la Ecuación 2 (NETO et al., 2016):

$$
\mathrm{E}_{\mathrm{oe}}=\mathrm{v}_{\mathrm{oe}}^{2} \rho_{\mathrm{CH}}
$$

Donde: $\mathrm{E}_{\mathrm{oe}}=$ Módulo dinámico $\left(\mathrm{N} \mathrm{m}^{-2}\right) ; \mathrm{v}_{\mathrm{oe}}=$ Velocidad de onda $\left(\mathrm{m} \mathrm{s}^{-1}\right) ; \rho_{\mathrm{CH}}=$ Densidad aparente a un contenido de humedad $\mathrm{CH}\left(\mathrm{kg} \mathrm{m}^{-3}\right)$.

La velocidad de onda medida y el módulo dinámico calculado se etiquetan con el subíndice “oe", para identificarlos como parámetros derivados de pruebas dinámicas de ondas de esfuerzo.

\section{Resultados y Discusión}

En la Tabla 1 se presentan la densidad aparente, la velocidad de onda y el módulo dinámico de la madera sólida y de la madera laminada de Enterolobium cyclocarpum, Tabebuia rosea y Juglans pyriformis. Se muestran, además, las variaciones aritméticas entre los resultados de la madera sólida y los de la madera laminada.

La densidad aparente de la madera laminada, en comparación con la de la madera sólida, se incrementa para Enterolobium cyclocarpum, Tabebuia rosea y Juglans pyriformis (Tabla 1). Asimismo, el coeficiente de variación aumenta para Tabebuia rosea y Juglans pyriformis. Sin embargo, disminuye para Enterolobium cyclocarpum. Si bien el tratamiento de laminado mejora la densidad aparente, incrementa la variabilidad para Tabebuia rosea.

La velocidad de onda en la madera laminada, en comparación con la de la madera sólida, disminuye para Enterolobium cyclocarpum, Tabebuia rosea y Juglans pyriformis (Tabla 1). Sin embargo, el coeficiente de variación aumenta para Enterolobium cyclocarpum. Así, el laminado de la madera reduce la velocidad de onda en la dirección longitudinal de las tres especies y disminuye la variabilidad para Tabebuia rosea y Juglans pyriformis.

El módulo dinámico de la madera laminada, en comparación con el de la madera sólida, aumenta para Enterolobium cyclocarpum y Tabebuia rosea. Sin embargo, disminuye para Juglans pyriformis (Tabla 1). En el mismo contexto, el coeficiente de variación disminuye para las tres especies. De manera particular a cada especie, el laminado de la madera aumenta el módulo dinámico y disminuye su variabilidad. 
Tabla 1. Densidad aparente, velocidad de onda y módulo dinámico de la madera sólida y de la madera laminada.

\begin{tabular}{|c|c|c|c|c|c|c|c|c|c|}
\hline & \multicolumn{3}{|c|}{ Madera sólida } & \multicolumn{3}{|c|}{ Madera laminada } & \multicolumn{3}{|c|}{ Diferencias } \\
\hline & $\begin{array}{c}\bullet_{\mathrm{CH}} \\
\left(\mathrm{kg} \mathrm{m}^{-3}\right) \\
\end{array}$ & $\begin{array}{c}\mathrm{v}_{\mathrm{oe}} \\
\left(\mathrm{m} \mathrm{s}^{-1}\right)\end{array}$ & $\begin{array}{c}\mathrm{E}_{\mathrm{oe}} \\
\left(\mathrm{MN} \mathrm{m}^{-2}\right)\end{array}$ & $\begin{array}{c}\rho_{\mathrm{CH}} \\
\left(\mathrm{kg} \mathrm{m}^{-3}\right)\end{array}$ & $\begin{array}{c}\mathrm{V}_{\mathrm{oe}} \\
\left(\mathrm{m} \mathrm{s}^{-1}\right)\end{array}$ & $\begin{array}{c}\mathrm{E}_{\mathrm{oe}} \\
\left(\mathrm{MN} \mathrm{m}^{-2}\right)\end{array}$ & $\begin{array}{c}\Delta \rho_{\mathrm{CH}} \\
(\%)\end{array}$ & $\begin{array}{c}\Delta \mathrm{v}_{\mathrm{oe}} \\
(\%)\end{array}$ & $\begin{array}{c}\Delta \mathrm{E}_{\mathrm{oe}} \\
(\%)\end{array}$ \\
\hline & \multicolumn{9}{|c|}{ Enterolobium. cyclocarpum } \\
\hline$\overline{\mathrm{x}}$ & 456 & 3225 & 4740 & 501 & 3133 & 4925 & $+9,9$ & $-2,9$ & $+3,9$ \\
\hline$\sigma$ & 47,2 & 125 & 565 & 38,4 & 148 & 575 & & & \\
\hline \multirow[t]{2}{*}{$\mathrm{CV}$} & 10,4 & 3,9 & 11,9 & 7,7 & 4,7 & 11,7 & $-26,0$ & $+20,5$ & $-1,7$ \\
\hline & \multicolumn{9}{|c|}{ Tabebuia rosea } \\
\hline$\overline{\mathrm{x}}$ & 621 & 3894 & 9407 & 693 & 3880 & 10442 & $+11,6$ & $-0,4$ & $+11,0$ \\
\hline$\sigma$ & 44,7 & 209 & 894 & 51,7 & 81 & 974 & & & \\
\hline \multirow[t]{2}{*}{$\mathrm{CV}$} & 7,2 & 5,4 & 9,5 & 7,5 & 2,1 & 9,3 & $+4,2$ & $-61,1$ & $-2,1$ \\
\hline & \multicolumn{9}{|c|}{ Juglans pyriformis } \\
\hline$\overline{\mathrm{x}}$ & 695 & 3496 & 8508 & 740 & 3349 & 8321 & $+6,5$ & $-4,2$ & $-2,2$ \\
\hline$\sigma$ & 57,5 & 199 & 1139 & 51,5 & 131 & 930 & & & \\
\hline $\mathrm{CV}$ & 8,3 & 5,7 & 13,4 & 7,0 & 3,9 & 11,2 & $-15,7$ & $-31,6$ & $-16,4$ \\
\hline
\end{tabular}

$\rho_{\mathrm{CH}}=$ Densidad aparente; $\mathrm{v}_{\mathrm{oe}}=$ Velocidad de onda; $\mathrm{E}_{\mathrm{oe}}=$ Módulo dinámico; $\overline{\mathrm{x}}=$ Media; $\sigma=$ Desviación estándar; $\mathrm{CV}=$ Coeficiente de variación porcentual; $\Delta=$ Variación aritmética entre madera sólida y madera laminada.

Utilizando el mismo aparato empleado en esta investigación, Bayestorff; Monteiro (2011) determinaron en madera laminada de Pinus taeda (Madera sólida: $\rho_{\mathrm{CH}}=448$ $\mathrm{kg} \mathrm{m} \mathrm{m}^{-3}$, madera laminada: $\rho_{\mathrm{CH}}=533 \mathrm{~kg} \mathrm{~m}^{-3}$ ), el módulo dinámico por ondas de esfuerzo de $8097 \mathrm{MN} \mathrm{m}^{-2} \mathrm{y}$ observaron un incremento de $17 \%$ en relación al módulo dinámico de la madera sólida de $6899 \mathrm{MN} \mathrm{m}^{-2}$ con la cual de elaboró la madera laminada. Este aumento en el módulo dinámico corresponde a un aumento en la densidad aparente de la madera laminada de $19 \%$.

Igualmente, Del Menezzi et al. (2013), determinaron en madera laminada de Schizolobium parahyba (Madera sólida: $\rho_{\mathrm{CH}}=392 \mathrm{~kg} \mathrm{~m}^{-3}$, madera laminada: $\left.\rho_{\mathrm{CH}}=467 \mathrm{~kg} \mathrm{~m}^{-3}\right)$, el módulo dinámico por ondas de esfuerzo de $9307 \mathrm{MN} \mathrm{m}^{-2,} \mathrm{y}$ observaron un incremento de $18 \%$ en relación al módulo dinámico de la madera sólida de $7837 \mathrm{MN} \mathrm{m}^{-2}$ con la cual se elaboró la madera laminada. Este aumento en el módulo dinámico corresponde a un aumento en la densidad aparente de la madera laminada de $19 \%$.

\section{Análisis estadístico}

La Tabla 2 presenta los resultados del análisis estadístico. La mayoría de las distribuciones de las muestras no fueron normales, lo que requirió realizar pruebas de comparación de medianas. Así, se observaron diferencias estadísticamente significativas prácticamente entre todas las muestras, con excepciones para el módulo dinámico de Enterolobium cyclocarpum y Juglans pyriformis.

Los resultados del análisis estadístico sugieren la intervención de factores que aumentaron la variabilidad de las magnitudes de las mediciones. Para verificar si el número de probetas observadas fue suficiente para validar el análisis estadístico, la Figura 2 presenta dos ejemplos de la convergencia del tamaño de la muestra de Enterolobium cyclocarpum: a) para madera sólida y b) para madera laminada. Los datos en los gráficos representan el número de probetas necesarias (n), en función del error de estimación aceptable (e), que es la magnitud relativa del parámetro en cuestión y que se acepta como error. 
Tabla 2. Resultados del análisis estadístico.

\begin{tabular}{cccccccc}
\hline & \multicolumn{2}{c}{ Madera sólida } & \multicolumn{2}{c}{ Madera laminada } & Ver-var & Anova & $\mathrm{K}-\mathrm{W}$ \\
\hline \multicolumn{1}{c}{$\rho_{\mathrm{CH}}$} & $\mathrm{SE}$ & $\mathrm{AE}$ & $\mathrm{SE}$ & $\mathrm{AE}$ & $\mathrm{P}$ & $\mathrm{P}$ & $\mathrm{P}$ \\
Enterolobium cyclocarpum & 1,754 & 0,854 & $-2,043$ & 2,222 & - & - & $<0,001^{*}$ \\
Tabebuia rosea & $-3,253$ & 3,570 & 4,202 & 4,615 & - & - & $<0,001^{*}$ \\
Juglans pyriformis & 3,625 & 2,853 & 2,508 & $-0,356$ & - & - & $0,002^{*}$ \\
\hline $\mathrm{V}_{\mathrm{oe}}$ & $\mathrm{SE}$ & $\mathrm{AE}$ & $\mathrm{SE}$ & $\mathrm{AE}$ & $\mathrm{P}$ & $\mathrm{P}$ & $\mathrm{P}$ \\
Enterolobium cyclocarpum & $-2,075$ & 2,201 & 1,756 & 3,219 & - & - & $0,005^{*}$ \\
Tabebuia rosea & $-2,795$ & 2,794 & 0,196 & $-0,318$ & - & - & $0,007^{*}$ \\
Juglans pyriformis & 0,579 & $-0,513$ & $-1,509$ & 0,446 & $0,033^{*}$ & - & $0,001^{*}$ \\
\hline $\mathrm{E}_{\mathrm{oe}}$ & $\mathrm{SE}$ & $\mathrm{AE}$ & $\mathrm{SE}$ & $\mathrm{AE}$ & $\mathrm{P}$ & $\mathrm{P}$ & $\mathrm{P}$ \\
Enterolobium cyclocarpum & 1,961 & 2,080 & 2,548 & 3,909 & - & - & 0,119 \\
Tabebuia rosea & $-3,536$ & 4,242 & 4,058 & 5,549 & - & - & $<0,001^{*}$ \\
Juglans pyriformis & 1,210 & $-0,52$ & 1,258 & 0,334 & 0,244 & 0,453 & - \\
\hline
\end{tabular}

$\rho_{\mathrm{CH}}=$ Densidad aparente; $\mathrm{v}_{\mathrm{oe}}=$ Velocidad de onda; $\mathrm{E}_{\mathrm{oe}}=$ Módulo dinámico; $\mathrm{SE}=$ Sesgo estandarizado; $\mathrm{AE}=\mathrm{Apuntamiento}$ estandarizado; ${ }^{\star} \mathrm{P}$ • 0.05: Existe una diferencia estadísticamente significativa.
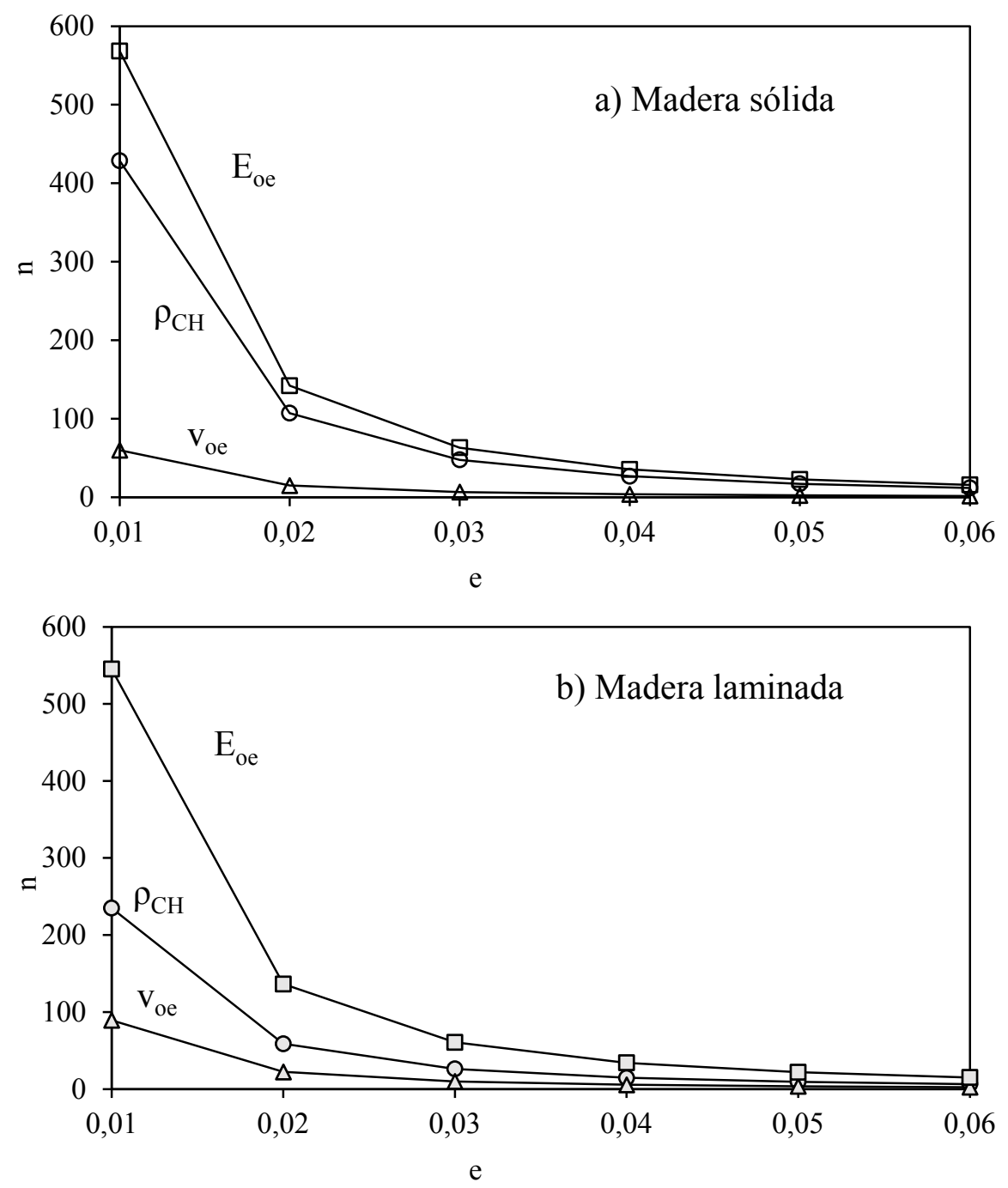

Figura 2. Convergencia del tamaño de la muestra (n), en función del error de estimación aceptable (e). Ejemplo para Enterolobium cyclocarpum. a) Madera sólida; b) Madera laminada. 
Los resultados de las convergencias para Enterolobium cyclocarpum (Figura 2), son similares a los obtenidos para Tabebuia rosea y Juglans pyriformis. La Tabla 3 presenta los resultados del cálculo a posteriori del tamaño de la muestra para la madera sólida y laminada de las tres especies para un error de estimación aceptable de 0,05. A pesar de la variabilidad en las mediciones, el tamaño de la muestra necesario para todas las muestras fue menor que 35 , cantidad de probetas observadas en esta investigación. Este resultado verifica que el tamaño de la muestra fue suficiente.

Las magnitudes en los resultados de $\rho_{\mathrm{CH}}, \mathrm{v}_{\mathrm{oe}} \mathrm{y} \mathrm{E}_{\mathrm{oe}}$ son similares a los datos reportados en la literatura para módulos de elasticidad determinados en condiciones estáticas (Tabla 4). Comparando los resultados de esta investigación con los de la bibliografía, se observa, por una parte, el aumento en la densidad aparente de la madera laminada en comparación con la densidad aparente de la madera sólida. Sin embargo, Komariah et al. (2015), reportan una disminución. Este resultado coincide con los de esta investigación donde la densidad aparente se incrementa para Juglans pyriformis y disminuye para Enterolobium cyclocarpum y Tabebuia rosea (Figura 3a). Por otra parte, se advierte que el módulo elástico aumenta y/o disminuye según la especie, resultado similar al de esta investigación donde el módulo dinámico aumenta para Juglans pyriformis y se reduce para Enterolobium cyclocarpum y Tabebuia rosea (Figura 3b). De tal forma, que es conveniente caracterizar el comportamiento mecánico de la madera laminada con un enfoque de experimentación de caso por caso de una especie en particular.

Tabla 3. Tamaño de la muestra.

\begin{tabular}{|c|c|c|c|c|c|c|}
\hline & \multicolumn{2}{|c|}{ Enterolobium cyclocarpum } & \multicolumn{2}{|c|}{ Tabebuia rosea } & \multicolumn{2}{|c|}{ Juilas pyriformis } \\
\hline & MS & ML & MS & ML & MS & ML \\
\hline & $(\mathrm{n})$ & $(\mathrm{n})$ & $(\mathrm{n})$ & (n) & (n) & $(\mathrm{n})$ \\
\hline$\rho_{\mathrm{CH}}$ & 17 & 9 & 8 & 9 & 11 & 8 \\
\hline $\mathrm{v}_{\mathrm{oe}}$ & 2 & 4 & 5 & 1 & 6 & 2 \\
\hline $\mathrm{E}_{\mathrm{oe}}$ & 23 & 22 & 14 & 10 & 29 & 20 \\
\hline
\end{tabular}

MS = Madera sólida; $M L=$ Madera laminada; $\rho_{\mathrm{CH}}=$ Densidad aparente $; \mathrm{v}_{\mathrm{oe}}=$ Velocidad de onda; $\mathrm{E}_{\mathrm{oe}}=$ Módulo dinámico. $\mathrm{n}=$ Tamaño de la muestra para un error de estimación de 0,05.

Tabla 4. Datos de la bibliografía.

\begin{tabular}{llcccccc}
\hline \multicolumn{1}{c}{ Referencia } & \multicolumn{1}{c}{ Especie } & \multicolumn{3}{c}{$\rho_{\mathrm{CH}}\left(\mathrm{kg} \mathrm{m}^{-3}\right)$} & \multicolumn{3}{c}{$\mathrm{MOE}\left(\mathrm{MN} \mathrm{m} \mathrm{m}^{-2}\right)$} \\
\cline { 3 - 8 } & & $\mathrm{MS}$ & $\mathrm{ML}$ & $\Delta$ & $\mathrm{MS}$ & $\mathrm{ML}$ & $\Delta$ \\
\hline 1 Araujo et al. (2005) & Enterolobium cyclocarpum & 350 & - & - & 47089 & 37288 & $-20,8$ \\
2 Araujo et al. (2005) & Bursera simaruba & 430 & - & - & 44210 & 42680 & $-3,5$ \\
3 Araujo et al. (2005) & Bucida buceras & 1020 & - & - & 104763 & 102877 & $-1,8$ \\
4 Erdil et al. (2009) & Fagusorientalis & 630 & 650 & 3,1 & 15503 & 10647 & $-31,3$ \\
5 Erdil et al. (2009) & Pinussylvestris & 460 & 540 & 14,8 & 8090 & 10889 & 34,6 \\
6 Erdil et al. (2009) & Populusnigra & 330 & 430 & 23,3 & 6304 & 7992 & 26,8 \\
7 Ribeiro et al. (2009) & Pinuspinaster & 550 & - & - & 7677 & 7801 & 1,6 \\
8 Nadir; Nagarajan (2014) & Hevea brasiliensis & 605 & - & - & 10448 & 9512 & $-9,0$ \\
9 Komariah et al. (2015) & Acacia mangium & 533 & 440 & $-21,1$ & 11930 & 8709 & $-27,0$ \\
10 Komariah et al. (2015) & Maesopsiseminii & 392 & 483 & 18,8 & 10166 & 9696 & $-4,6$ \\
11 Komariah et al. (2015) & Falcatariamoluccana & 271 & 290 & 6,6 & 6037 & 6209 & 2,8 \\
12 Gaborik et al. (2016) & Populusnigra & 330 & - & - & 11654 & 10102 & $-13,3$ \\
\hline
\end{tabular}

$\rho_{\mathrm{CH}}=$ Densidad aparente; $\mathrm{MOE}=$ Módulo de elasticidad; $\mathrm{MS}=$ Madera sólida; $\mathrm{ML}=$ Madera laminada; $\Delta=$ Variación aritmética entre madera sólida y madera laminada (\%). 

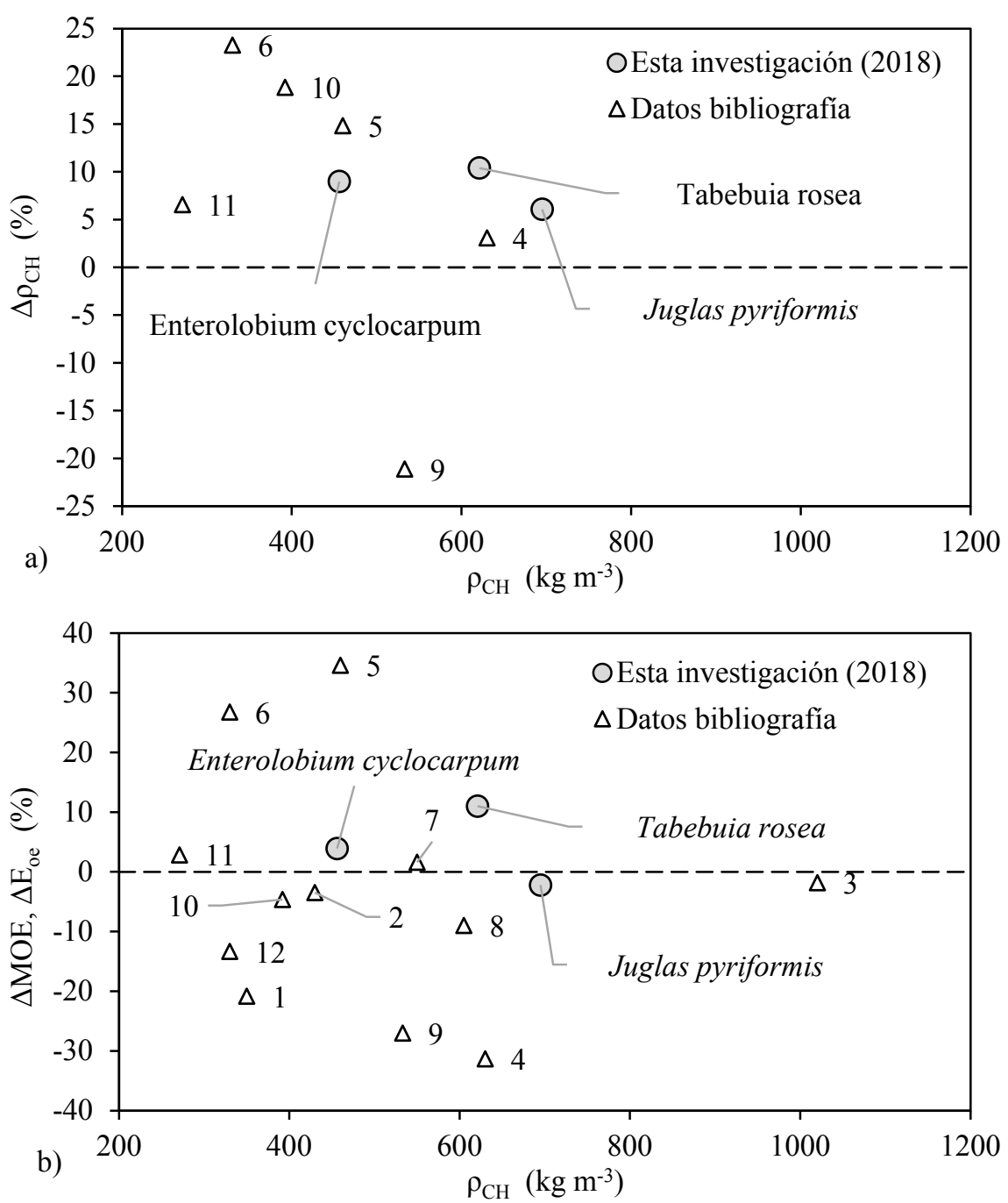

Figura 3. Dispersión de a) la variación de la densidad $\left(\Delta \rho_{\mathrm{CH}}\right)$ y b), variación de los módulos de elasticidad $(\Delta \mathrm{MOE}), \mathrm{y}$ dinámico $\left(\Delta \mathrm{E}_{\mathrm{oe}}\right)$, en función de la densidad aparente $\left(\rho_{\mathrm{CH}}\right)$. La numeración corresponde a la Tabla 4.

\section{Conclusiones}

El tratamiento de laminado de la madera de Enterolobium cyclocarpum, Tabebuia rosea y Juglans pyriformis incrementa su densidad aparente y disminuye su velocidad de onda. Por su parte, los módulos dinámicos de Enterolobium cyclocarpum y Tabebuia rosea aumentan, pero decrece para Juglans pyriformis. En el mismo contexto, la variabilidad de la densidad aparente, la velocidad de onda y el módulo dinámico, aumenta o disminuye según se trate de la especie y/o del parámetro en cuestión.

Las magnitudes de los valores promedio de las densidades aparentes, de las velocidades de onda y de los módulos dinámicos, se sitúan en los intervalos reportados en la bibliografía para las especies mexicanas. Comparativamente, sus valores son similares a las de las referencias internacionales.

Los resultados de esta investigación son válidos para el caso de laminado de pequeñas probetas de las especies estudiadas. Para su aplicación a proyectos de ingeniería, donde las dimensiones de los elementos en cuestión son de grandes dimensiones, es recomendable realizar estudios ex profeso. 


\section{Agradecimientos}

La investigación estuvo patrocinada por la Coordinación de la Investigación Científica, de la Universidad Michoacana de San Nicolás de hidalgo, México.

\section{Referencias}

ARAUJO MOLINA, O.; CERÓN CARDEÑA, M.; CHAN MARTÍN, M.; AZUETA GARCÍA, M. Resistencia a la flexión de vigas laminadas con tres especies de madera tropical mexicana. Ingeniería, v. 9, n. 1, p. 5-12, 2005.

BAYESTORFF DA CUNHA, A.; MONTEIRO DE MATOS, J. L. Estimativa do módulo de elasticidade em vigas laminadas coladas pelos métodos estático e dinâmico. Floresta, v. 41, n. 1, p. 97-112, 2011.

BOURREAU, D.; AIMENE, Y.; BEAUCHÊNE, J.; THIBAUT, B. Feasibility of glued laminated timber beams with tropical hardwoods. European Journal of Wood and Wood Products, v. 71, n. 5, p. 653-662, 2013.

CORDERO, J.; BOSHIER, D. H. Árboles de Centroamérica. San José: Centro Agronómico Tropical de Investigación y Enseñanza, 2003.

DE SOUZA, F; DEL MENEZZI, C., BORTOLOTTO JÚNIOR, G. Material properties and nondestructive evaluation of laminated veneer lumber (LVL) made from Pinus oocarpa and P. kesiya. European Journal of Wood and Wood Products, v. 69, n. 2, p. 183-192, 2011.

DEL MENEZZI, C.; MENDES, L.; DE SOUZA, M.; BORTOLETTO JR, G. Effect of nondestructive evaluation of veneers on the properties of laminated veneer lumber (LVL) from a tropical species. Forests, v. 4, n. 2, p. 270-278, 2013.

ERDIL, Y. Z.; KASAL, A.; ZHANG, J.; EFE, H.; DIZEI, T. Comparison of mechanical properties of solid wood and laminated veneer lumber fabricated from Turkish beech, Scotch pine, and Lombardy poplar. Forest Products Journal, v. 59 , n. 6 , p. 55-60, 2009.

GABORIK, J.; GAFF, M.; RUMAN, D.; ZABORSKY, V.; KASICKOVA, V.; SIKORA, A. Adhesive as a Factor Affecting the Properties of Laminated Wood. Bioresources, v. 11, n. 4, p. 10565-10574, 2016.
GUTIÉRREZ PULIDO, H.; DE LA VARA SALAZAR, R. Análisis y diseño de experimentos. México: McGraw Hill, 2012.

INTERNATIONAL ORGANIZATION FOR STANDARDIZATION. ISO 13061-1:2014. Wood. Determination of moisture content for physical and mechanical tests. Geneva: International Organization for Standardization, 2014a.

INTERNATIONAL

ORGANIZATION

FOR

STANDARDIZATION. ISO 3129:2012. Wood. Sampling methods and general requirements for physical and mechanical tests. Geneva: International Organization for Standardization, 2012.

INTERNATIONAL ORGANIZATION FOR STANDARDIZATION. ISO 13061-2:2014. Wood. Determination of density for physical and mechanical tests. Geneva: International Organization for Standardization, 2014b.

KOMARIAH, R. N.; HADI, Y. S.; MASSIJAYA, M. Y.; SURYANA, J. Physical-mechanical properties of glued laminated timber made from tropical small-diameter logs grown in Indonesia. Journal of the Korean Wood Science and Technology, v. 43, n. 2, p. 156-167, 2015.

MANSFIELD, S. D.; PARISH, OTT, P. K.; HART, J. F.; GOUDIE, J. W. Assessing the wood quality of interior spruce (Piceaglauca $\times$ P. engelmannii): variation in strength, relative density, microfibril angle, and fiber length. Holzforschung, v. 70, n. 3, p. 223-234, 2016.

NADIR, Y.; NAGARAJAN, P. The behavior of horizontally glued laminated beams using rubber wood. Construction and Building Materials, v. 55, p. 398-405, 2014.

NETO, P. N. M.; PAES, J. B.; SEGUNDINHO, P. G. D. Evaluation of elasticity and rupture modulus of woods by destructive and non-destructive techniques. Scientia Forestalis, v. 44, n. 111, p. 683-690, 2016.

NIKLAS, K. J.; SPATZ. H. C. Worldwide correlations of mechanical properties and green wood density. American Journal of Botany. v. 97, n. 10, p. 1587-1594, 2010.

RIBEIRO, A. S.; DE JESUS, A. M. P.; LIMA, A. M.; LOUSADA, J. L. C. Study of strengthening solutions for glued laminated wood beams of maritime pine wood. Construction and Building Materials, v. 23, n. 8, p. 27382745, 2009. 
SANDBERG, D.; KUTNAR, A.; MANTANIS, G. Wood modification technologies. A review. iForest-Biogeosciences and Forestry, v. 10, n. 6, p. 895-908, 2017.

SILVA GUZMÁN, J. A., FUENTES TALAVERA, F. J., RODRÍGUEZ ANDA R., TORRES ANDRADE P. A., LOMELÍ RAMÍREZ M. A., RAMOS QUIRARTE J., WAITKUS C., \& RICHTER, H. G. Fichas de propiedades tecnológicas y usos de maderas nativas de México e importadas. México: Comisión Nacional Forestal, 2010.

SOTOMAYOR CASTELLANOS, J. R. Banco FITECMA de características físico-mecánicas de maderas mexicanas. Morelia: Universidad Michoacana de San Nicolás de Hidalgo, 2015.

SOTOMAYOR CASTELLANOS., J. R.; F. RUIZ AQUINO, F. Caracterización dinámica de madera laminada. Investigación e Ingeniería de la Madera, v. 13, n. 3, p. 22-44, 2017.

TAMARIT URIAS, J. C.; LÓPEZ TORRES J. L. Xilotecnología de los principales árboles tropicales de México. México: Instituto Nacional de Investigaciones Forestales, Agrícolas y Pecuarias, 2007.

WALKER, J. C. F. Primary Wood Processing. Dordrecht: Springer, 2006.

WILLIAMSON, G. B.; WIEMANN, M. C. Measuring wood specific gravity...Correctly. American Journal of Botany, v. 97, n.3, p. 519-524, 2010.

WILLIAMSON, T. G. APA Engineered Wood Handbook. New York: McGraw-Hill, 2001. 